\title{
TOXICIDADE RESIDUAL DE ALGUNS AGROTÓXICOS RECOMENDADOS NA CITRICULTURA SOBRE Neoseiulus californicus (McGREGOR) (ACARI: PHYTOSEIIDAE) ${ }^{1}$
}

\author{
MARCOS ZATTIDA SILVA² \& CARLOS AMADEU LEITE DE OLIVEIRA ${ }^{3}$
}

RESUMO - O objetivo do trabalho foi avaliar a toxicidade residual de alguns agrotóxicos utilizados em citros sobre Neoseiulus californicus (McGregor) em condições de laboratório. O método de bioensaio adotado foi o de contato residual. Folhas de citros da variedade Pêra, acondicionadas em arenas, foram pulverizadas em torre de Potter. A toxicidade residual dos produtos foi avaliada duas horas e 1;3; 5; 7; 10; 14 e 21 dias após a aplicação. Em cada arena, foram transferidas dez fêmeas adultas de $N$. californicus, juntamente com uma quantidade suficiente de Tetranychus urticae, como fonte de alimento. As avaliações de mortalidade foram realizadas 72 horas após a transferência dos ácaros para as arenas. Os agrotóxicos acrinathrin, deltamethrin, dinocap, enxofre, fenpropathrin, óxido de fenbutatin e propargite não causaram mortalidades significativas em adultos de $N$. californicus. Foram registradas mortalidades de 29,8; 24,0 e 34,1\% para ácaros $N$. californicus expostos a resíduos de duas horas de idade de abamectim, azocyclotin e cyhexatin, respectivamente. Dicofol, pyridaben e chlorfenapyr causaram $100 \%$ de mortalidade aos ácaros predadores expostos aos resíduos tóxicos dos acaricidas, com duas horas de idade. Abamectin provocou mortalidade significativa por um período inferior a um dia. Resíduos dos acaricidas azocyclotin, cyhexatin, dicofol, pyridaben e chlorfenapyr provocaram mortalidades significativas por períodos de $1 ; 1 ; 10 ; 10$ e 21 dias, respectivamente. Os resultados obtidos no presente experimento servem de subsídio para a escolha adequada dos agrotóxicos a serem utilizados em pomares de citros nos quais $N$. californicus esteja presente ou naqueles em que o predador venha a ser liberado. Esses resultados também servem para a escolha do momento mais favorável para a liberação dos ácaros predadores dessa espécie no campo, após a aplicação de agrotóxicos nos pomares. Estudos conduzidos em condições de campo ainda são necessários para se compreender melhor o efeito desses agrotóxicos sobre o ácaro predador.

Termos para indexação: Ácaro predador, controle biológico, sensibilidade a agrotóxicos.

\section{RESIDUAL TOXICITY OF SOME PESTICIDES RECOMMENDED FOR CITRUS ORCHARDS ON THE PREDACEOUS MITE Neoseiulus californicus (McGREGOR) (ACARI: PHYTOSEIIDAE)}

\begin{abstract}
This study was carried out to evaluate the residual toxicity of some pesticides used in citrus orchards, on Neoseiulus californicus (McGregor) under laboratory conditions. The residual contact bioassay method was adopted. Citrus leaves of the variety "Pêra" were sprayed in a Potter tower. The products' residual toxicity was evaluated at two hours and 1, 3, 5, 7, 10, 14 and 21 days after treatment. Ten adult females of $N$. californicus were transferred to each arena together with an enough amount of Tetranychus urticae to feed the predator. Mortality evaluations were performed at 72 hours after transferring the predaceous mites to the arenas. The pesticides acrinathrin, deltamethrin, dinocap, sulphur, fenpropathrin, fenbutatin oxide and propargite did not cause significant mortalities to the adults of $N$. californicus. Abamectim, azocyclotin and cyhexatin caused mortalities of 29.8, 24.0 and 34.1\%, respectively, for $N$. californicus adults exposed to two-hour pesticide residues. Dicofol, pyridaben and chlorfenapyr caused $100 \%$ of mortality to the predators exposed to the two-hour acaricide residues. Abamectin provoked significant mortalities for a period shorter than one day. Residues of azocyclotin, cyhexatin, dicofol, pyridaben and chlorfenapyr caused significant mortalities for periods of $1,1,10,10$ and 21 days, respectively. The results of this study provided basic information for choosing the adequate pesticides to be used in citrus orchards in which $N$. californicus is present, or in those the predator will be released. The results are also useful for the decision of the best releasing time for $N$. californicus in the field, after pesticide applications. Studies carried out in the field are still necessary to understand better the effect of these pesticides under the predaceous mite.
\end{abstract}

Index terms: Predatory mite, citrus, biological control, sensibility to agrochemicals.

\section{INTRODUÇÃO}

Embora o período de toxicidade residual da maioria dos acaricidas utilizados em citros seja limitado a alguns dias ou semanas, o tempo necessário para o restabelecimento da população de ácaros predadores, após a aplicação de acaricidas, em condições de campo, tem se mostrado relativamente longo (Sato \& Raga, 1998). Neste contexto, o conhecimento do período

\footnotetext{
1 (Trabalho 054-2006). Recebido em 28-04-2006. Aceito para publicação em 08-12-2006.

2 Pós-graduando em Agronomia-Entomologia Agrícola, FCAV-UNESP. makdsil@ig.com.br

${ }^{3}$ Prof. Titular Depto.de Fitossanidade,.FCAV/UNESP.amadeu@fcav.unesp.br
} 
de toxicidade residual dos agrotóxicos serve de subsídio para programas de manejo de ácaros-praga em citros, quando se pretende realizar a liberação de ácaros predadores visando ao controle biológico de ácaros fitófagos na cultura.

O controle biológico exercido pelos inimigos naturais é o principal componente do manejo integrado de pragas e responsável pelo seu sucesso (Yamamoto et al., 1995). Diversos estudos sobre manejo do ácaro da leprose Brevipalpus phoenicis (Geijskes) têm enfocado a preservação dos inimigos naturais pela utilização de produtos seletivos (Komatsu \& Nakano, 1988; Sato et al., 1995; Reis et al., 1999). Todavia, o uso indiscriminado dos agrotóxicos sobre os organismos benéficos tem provocado problemas em diversas culturas, como a resistência de insetos e ácaros a agrotóxicos, a ressurgência de pragas e intenso desequilíbrio ecológico (Van de Vrie et al., 1972).

Dentre os predadores presentes em citros, aqueles pertencentes à família Phytoseiidae são os mais importantes (McMurtry et al., 1970), sendo cerca de 2.250 espécies acarinas descritas mundialmente (Moraes et al., 2004), das quais aproximadamente 200 já foram observadas no Brasil (Moraes, 1992, Moraes et al., 2004). Neoseiulus californicus (McGregor) é um ácaro predador da família Phytoseiidae que promove o controle biológico de ácaros tetraniquídeos em várias plantas cultivadas, como morango, maçã, feijão, ornamentais, etc. (Moraes et al., 1986). Este fitoseídeo ocorre nas regiões semitropicais e temperadas da América do Sul, e também nas áreas áridas do sul da Califórnia e sul da Europa (McMurtry \& Croft, 1997).

No Brasil, $N$. californicus tem sido criado massalmente e liberado em macieira no Sul do País, visando ao controle de Panonychus ulmi Koch (Monteiro, 1994). O ácaro tem se mostrado um inimigo natural bastante promissor, permitindo uma redução significativa no uso de acaricidas. Em algumas áreas, foi possível o controle de $P$. ulmi apenas com a utilização de $N$. californicus, sem a necessidade da aplicação de acaricidas a partir do segundo ano após o início das liberações do ácaro predador (Monteiro, 2002). A importância desse predador em citros ainda é desconhecida no Brasil.

Dessa forma, pretende-se, com este estudo, obter informações que contribuam para uma futura utilização desse predador num programa de manejo de ácaros-praga em citros, por meio de sua liberação. $\mathrm{O}$ objetivo do presente trabalho foi avaliar a toxicidade residual de alguns dos principais agrotóxicos utilizados em citros, no Brasil, sobre uma população de $N$. californicus e obter informações de produtos que não causem mortalidade significativa desse predador.

\section{MATERIAL E MÉTODOS}

A pesquisa foi realizada no Laboratório de Acarologia do Departamento de Fitossanidade da Faculdade de Ciências Agrárias e Veterinária, da Universidade Estadual Paulista, Câmpus de Jaboticabal - SP.

A população de $N$. californicus, cedida pelo Dr. Mário Eidi Sato, do Laboratório de Entomologia Econômica do Centro Experimental Central, do Instituto Biológico, Campinas - SP, foi coletada em cultivo comercial de morangueiro, no município de Atibaia-SP, em 30-10-1999. A identificação desses ácaros foi realizada pelo Prof. Dr. Gilberto José de Moraes, da Escola Superior de Agricultura "Luiz de Queiroz" / USP, Piracicaba-SP.

Criação do predador - A partir dessa coleta, mantevese uma criação-estoque de $N$. californicus no laboratório sobre plantas de feijão-de-porco [Canavalia ensiformis (L.)] cultivadas em vasos plásticos de $500 \mathrm{~mL}$, tendo como fonte alimentar Tetranychus urticae Koch, sendo, posteriormente, transferidos para discos de folha de laranjeira da variedade Pêra, com aproximadamente $8 \mathrm{~cm}$ de diâmetro, colocados sobre camada de algodão hidrófilo mantida saturada com água destilada em placa de Petri (15 cm de diâmetro). A finalidade da transferência dos predadores para arenas de folha de laranjeira foi facilitar a condução dos experimentos em laboratório. Nesse aspecto, os ácaros fitoseídeos criados em arenas de folhas de citros podem ser encontrados e coletados muito mais facilmente do que quando eles são criados em plantas de feijão.

Para evitar a fuga de ácaros, manteve-se a borda da folha coberta com algodão umedecido. Ninfas e adultos de $T$. urticae e pólen de mamona, Ricinus communis L., foram colocados em abundância, em cada arena, para servir de alimento aos ácaros predadores. A criação foi mantida em sala climatizada, a $25 \pm 2{ }^{\circ} \mathrm{C}$, umidade relativa de $70 \pm 10 \%$ e fotofase de $12 \mathrm{~h}$.

Testes toxicológicos - Os agrotóxicos testados foram empregados nas concentrações recomendadas para o controle do ácaro da leprose B. phoenicis. Deltamethrin (inseticida) foi utilizado na concentração indicada para o controle de diversos insetos-praga em citros (Andrei, 1999) (Tabela 1).

Toxicidade residual em $N$. californicus - Os testes com os agrotóxicos foram realizados com base no método descrito por Knight et al. (1990). Discos de folha de laranjeira (variedade Pêra), de $4 \mathrm{~cm}$ de diâmetro, foram colocados sobre uma camada de algodão hidrófilo, em uma placa de Petri ( $9 \mathrm{~cm}$ de diâmetro). A camada de algodão foi mantida sempre saturada com água destilada. A borda da folha também foi coberta por algodão umedecido, formando uma barreira para evitar a fuga dos ácaros. Adotou-se o delineamento experimental inteiramente casualisado, onde cada tratamento foi repetido cinco vezes (cinco arenas com dez ácaros predadores).

A pulverização dos produtos (Tabela 1) foi realizada em torre de Potter (Burkard Scientific, Uxbridge, UK), aplicando um volume de $2 \mathrm{ml}$ de calda, à pressão de $0,703 \mathrm{~kg} / \mathrm{cm}^{2}$, o que correspondeu a um depósito de aproximadamente $1,5 \mathrm{mg}$ de calda por $\mathrm{cm}^{2}$ de folha. Decorridas duas horas e $1 ; 3 ; 5 ; 7 ; 10 ; 14$ e 21 dias da aplicação, efetuou-se a transferência de 10 fêmeas adultas de $N$. californicus para cada arena. Ninfas e adultos de T. urticae foram fornecidos, em abundância, como alimento aos ácaros predadores. $\mathrm{O}$ experimento foi mantido em sala climatizada, a 25 $\pm 2{ }^{\circ} \mathrm{C}$, umidade relativa de $70 \pm 10 \%$ e fotofase de $12 \mathrm{~h}$.

Para a condução deste experimento, foi necessário preparar um número elevado de arenas para avaliar atividade biológica de resíduo de agrotóxicos de até 21 dias. Apenas para a realização da avaliação do efeito do resíduo de chlorfenapyr com 21 dias de idade, foram preparadas 25 arenas, sendo aproveitadas apenas cinco arenas com folhas em bom estado de conservação.

As avaliações do número de ácaros vivos e mortos foram realizadas 72 horas após a transferência para as arenas tratadas, 
com auxílio de um microscópio estereoscópico. Consideraramse mortos os ácaros predadores que não conseguiam locomoverse por uma distância mínima equivalente ao comprimento do seu corpo, ao serem tocados levemente com pincel de pêlos macios.

O número de ácaros vivos, por parcela, foi transformado em $\sqrt{x+0,5}$ e submetido à analise de variância, pelo teste $F$, e as médias, comparadas pelo teste Tukey, a $5 \%$ de probabilidade. Os percentuais de redução populacional foram calculados pela fórmula de Henderson \& Tilton (1955).

\section{RESULTADOS E DISCUSSÃO}

Toxicidade residual em $\boldsymbol{N}$. californicus - O estudo da toxicidade residual de agrotóxicos permite estabelecer o período necessário a ser respeitado após a aplicação de um determinado produto químico para a liberação de um inimigo natural na cultura, para evitar a intoxicação do predador. A preservação dos inimigos naturais é uma das práticas de maior importância no manejo integrado de pragas, contribuindo para manter o nível de injúria abaixo do tolerável.

Nos bioensaios de toxicidade residual (Tabela 2), os agrotóxicos: acrinathrin, deltamethrin, dinocap, enxofre, fenpropathrin, óxido de fenbutatin e propargite não causaram mortalidades significativas a $N$. californicus, não diferindo estatisticamente $(P>0,05)$ da testemunha. Embora os piretróides acrinathrin, deltamethrin e fenpropathrin não tenham se mostrado prejudiciais ao fitoseídeo no presente trabalho, esses produtos têm apresentado elevada toxicidade a outras espécies de ácaros predadores dessa família (Malezieux et al., 1992; Yamamoto et al., 1992; Sato et al., 1996; Reis et al., 1998). A alta toxicidade de piretróides a fitoseídeos de outras espécies também foi mencionada em diversos trabalhos (Croft \& Whalon, 1982; Zacharda \& Hlùchy, 1991; Yamamoto et al. 1992).
Com relação aos acaricidas, propargite (Sato et al., 1996; Morse et al., 1987; Reis et al., 1998) e enxofre (Reis et al., 1998), diferentemente do constatado no presente trabalho (Tabela 3), as citações reportam a alta toxicidade desses produtos a outras espécies de fitoseídeos, presentes em citros.

$\mathrm{Na}$ avaliação realizada 72 horas após a transferência dos ácaros para arenas de folha de citros com idade de resíduo de agrotóxico de duas horas, verificou-se que enxofre, óxido de fenbutatin, propargite, fenpropathrin, dinocap, deltamethrin e acrinathrin não causaram mortalidades significativa a adultos de $N$. californicus (Tabelas 2 e 3 ). Abamectin, azocyclotin e cyhexatin acarretaram mortalidades de $29,8 \% ; 24,0 \%$ e $34,1 \%$, respectivamente, 72 horas após a transferência dos ácaros para arenas de folha de citros com idade de resíduo de agrotóxicos de duas horas.

Os agrotóxicos chlorfenapyr, dicofol e pyridaben mostraram-se nocivos a $N$. californicus, causando $100 \%$ de mortalidade, 72 horas após a transferência dos ácaros para arenas de folha de citros com idade de resíduo de agrotóxicos de duas horas (Tabela 2).

Para abamectin, mortalidades significativas de adultos de $N$. californicus foram observadas apenas para ácaros transferidos para arenas com idade de resíduo de duas horas. Nas avaliações posteriores, não foi observado efeito tóxico significativo do produto (Tabelas 2 e 3). Resultados semelhantes com abamectin foram observados por Sato et al. (1996), em citros, cujo período de toxicidade residual para Iphyseiodes zuluagai Denmark \& Muma foi de apenas um dia, com mortalidade inicial inferior a $62 \%$.

Verifica-se que a mortalidade dos ácaros transferidos para folhas com três e cinco dias após a aplicação continuou elevada apenas nos tratamentos com chlorfenapyr, dicofol e pyridaben. Para os demais, as mortalidades foram iguais ou inferiores a 6,9\% (Tabela 3), com número médio de ácaros vivos semelhantes ao da testemunha (Tabela 2)

TABELA 1 - Relação de produtos testados (nome técnico, nome comercial e formulação, dosagem do produto comercial e do ingrediente ativo, classificação quanto ao uso e grupo químico), recomendados para pragas de citros no Brasil.

\begin{tabular}{|c|c|c|c|c|c|}
\hline $\begin{array}{l}\text { Nome } \\
\text { Técnico }\end{array}$ & $\begin{array}{c}\text { Nome Comercial } \\
\mathrm{e} \\
\text { Formulação }\end{array}$ & $\begin{array}{l}\text { Dosagem. } \\
\text { g ou ml p.c. } \\
100 \mathrm{~L}\end{array}$ & $\begin{array}{c}\text { Dosagem } \\
\text { g de i. a } \\
100 \mathrm{~L}\end{array}$ & Uso $^{1}$ & Grupo químico \\
\hline abamectin & Vertimec CE 18 & 30,0 & 0,54 & $\mathrm{~A}$ & Avermectina \\
\hline acrinathrin & Rufast 50 SC & 10,0 & 0,50 & A & Piretróide \\
\hline azocyclotin & Caligur $500 \mathrm{SC}$ & 50,0 & 25,00 & A & Organoestânico \\
\hline cyhexatin & Sipcatin $500 \mathrm{SC}$ & 50,0 & 25,00 & $\mathrm{~A}$ & Organoestânico \\
\hline chlorfenapyr & Citrex 240 SC & 62,5 & 15,00 & $\mathrm{I}, \mathrm{A}$ & Análago de pirazol \\
\hline deltamethrin & Decis $25 \mathrm{CE}$ & 50,0 & 1,25 & I & Piretróide \\
\hline dicofol & Kelthane $480 \mathrm{CE}$ & 77,0 & 37,00 & A & Organoclorado \\
\hline dinocap & Karathane 369 CE & 50,0 & 18,45 & A & Dinitrofenol \\
\hline enxofre & Thiovit 80 PM & 500,0 & 400,00 & $\mathrm{~F}, \mathrm{~A}$ & Inorgânico \\
\hline fenpropathrin & Danimen $300 \mathrm{CE}$ & 50,0 & 15,00 & $\mathrm{I}, \mathrm{A}$ & Piretróide \\
\hline óxido de fenbutatin & Torque $500 \mathrm{SC}$ & 80,0 & 40,00 & $\mathrm{~A}$ & Organoestânico \\
\hline propargite & Omite $720 \mathrm{CE}$ & 100,0 & 72,00 & A & Sulfito de alquila \\
\hline pyridaben & Sanmite $200 \mathrm{CE}$ & 75,0 & 15,00 & A & Piridazinona \\
\hline
\end{tabular}


TABELA 2 - Número médio de ácaros vivos (Neoseiulus californicus), expostos por 72h sobre folhas de citros, em diferentes períodos, após o tratamento.

\begin{tabular}{|c|c|c|c|c|c|c|c|c|c|}
\hline \multirow{2}{*}{ Tratamentos } & \multirow{2}{*}{$\begin{array}{c}\text { Dosag. } \\
\text { g de i.a. } \\
100 L\end{array}$} & \multicolumn{8}{|c|}{ Períodos da aplicação à transferência } \\
\hline & & 2 horas & 1 dia & 3 dias & 5 dias & 7 dias & 10 dias & 14 dias & 21 dias \\
\hline abamectin & 0,54 & $6,60 * \pm 0,24 \mathrm{c}$ & $9,40 \pm 0,40 \mathrm{ab}$ & $10,00 \pm 0,00 \mathrm{a}$ & $10,00 \pm 0,00 \mathrm{a}$ & $10,00 \pm 0,00 \mathrm{a}$ & $10,00 \pm 0,00 \mathrm{a}$ & $10,00 \pm 0,00 \mathrm{a}$ & $10,00 \pm 0,0 \mathrm{a}$ \\
\hline acrinathrin & 0,50 & $9,20 \pm 0,37 \mathrm{ab}$ & $9,20 \pm 0,37 \mathrm{ab}$ & $10,00 \pm 0,00 \mathrm{a}$ & $10,00 \pm 0,00 \mathrm{a}$ & $10,00 \pm 0,00 \mathrm{a}$ & $10,00 \pm 0,00 \mathrm{a}$ & $10,00 \pm 0,00 \mathrm{a}$ & $10,00 \pm 0,0 \mathrm{a}$ \\
\hline azocyclotin & 25,00 & $7,60 \pm 0,40 \mathrm{bc}$ & $6,80 \pm 0,20 \mathrm{c}$ & $9,80 \pm 0,20 \mathrm{a}$ & $10,00 \pm 0,00 \mathrm{a}$ & $10,00 \pm 0,00 \mathrm{a}$ & $10,00 \pm 0,00 \mathrm{a}$ & $10,00 \pm 0,00 \mathrm{a}$ & $10,00 \pm 0,0 \mathrm{a}$ \\
\hline cyhexatin & 25,00 & $6,20 \pm 0,58 \mathrm{c}$ & $7,80 \pm 0,37 \mathrm{bc}$ & $8,20 \pm 0,66 \mathrm{a}$ & $9,80 \pm 0,20 \mathrm{a}$ & $10,00 \pm 0,00 \mathrm{a}$ & $10,00 \pm 0,00 \mathrm{a}$ & $10,00 \pm 0,00 \mathrm{a}$ & $10,00 \pm 0,0 \mathrm{a}$ \\
\hline clorfenapyr & 15,00 & $0,00 \pm 0,00 \mathrm{~d}$ & $0,40 \pm 0,24 d$ & $0,40 \pm 0,24 b$ & $0,40 \pm 0,31 \mathrm{c}$ & $1,20 \pm 0,37 d$ & $2,20 \pm 0,31 \mathrm{c}$ & $5,60 \pm 1,89 \mathrm{c}$ & $8,60 \pm 0,0 \mathrm{~b}$ \\
\hline deltamethrin & 1,25 & $9,20 \pm 0,37 \mathrm{ab}$ & $9,20 \pm 0,20 \mathrm{ab}$ & $10,00 \pm 0,00 \mathrm{a}$ & $10,00 \pm 0,00 \mathrm{a}$ & $10,00 \pm 0,00 \mathrm{a}$ & $10,00 \pm 0,00 \mathrm{a}$ & $10,00 \pm 0,00 \mathrm{a}$ & $10,00 \pm 0,0 \mathrm{a}$ \\
\hline cyhexatin & 25,00 & $6,20 \pm 0,58 c$ & $7,80 \pm 0,37 \mathrm{bc}$ & $8,20 \pm 0,66 \mathrm{a}$ & $9,80 \pm 0,20 \mathrm{a}$ & $10,00 \pm 0,00 \mathrm{a}$ & $10,00 \pm 0,00 \mathrm{a}$ & $10,00 \pm 0,00 \mathrm{a}$ & $10,00 \pm 0,0 \mathrm{a}$ \\
\hline clorfenapyr & 15,00 & $0,00 \pm 0,00 \mathrm{~d}$ & $0,40 \pm 0,24 \mathrm{~d}$ & $0,40 \pm 0,24 b$ & $0,40 \pm 0,31 \mathrm{c}$ & $1,20 \pm 0,37 \mathrm{~d}$ & $2,20 \pm 0,31 \mathrm{c}$ & $5,60 \pm 1,89 \mathrm{c}$ & $8,60 \pm 0,0 \mathrm{~b}$ \\
\hline deltamethrin & 1,25 & $9,20 \pm 0,37 \mathrm{ab}$ & $9,20 \pm 0,20 \mathrm{ab}$ & $10,00 \pm 0,00 \mathrm{a}$ & $10,00 \pm 0,00 \mathrm{a}$ & $10,00 \pm 0,00 \mathrm{a}$ & $10,00 \pm 0,00 \mathrm{a}$ & $10,00 \pm 0,00 \mathrm{a}$ & $10,00 \pm 0,0 \mathrm{a}$ \\
\hline dicofol & 37,00 & $0,00 \pm 0,00 \mathrm{~d}$ & $0,60 \pm 0,24 \mathrm{~d}$ & $0,80 \pm 0,58 b$ & $3,20 \pm 0,73 b$ & $7,60 \pm 0,51 b$ & $7,80 \pm 0,94 b$ & $9,80 \pm 0,00 \mathrm{ab}$ & $10,00 \pm 0,0 \mathrm{a}$ \\
\hline dinocap & 18,45 & $9,40 \pm 0,40 \mathrm{ab}$ & $9,40 \pm 0,37 \mathrm{ab}$ & $9,60 \pm 0,24 \mathrm{a}$ & $10,00 \pm 0,00 \mathrm{a}$ & $10,00 \pm 0,00 \mathrm{a}$ & $10,00 \pm 0,00 \mathrm{a}$ & $10,00 \pm 0,00 \mathrm{a}$ & $10,00 \pm 0,0 \mathrm{a}$ \\
\hline enxofre & 400,00 & $9,60 \pm 0,40 \mathrm{a}$ & $9,80 \pm 0,20 \mathrm{a}$ & $10,00 \pm 0,00 \mathrm{a}$ & $10,00 \pm 0,00 \mathrm{a}$ & $10,00 \pm 0,00 \mathrm{a}$ & $10,00 \pm 0,00 \mathrm{a}$ & $10,00 \pm 0,00 \mathrm{a}$ & $10,00 \pm 0,0 \mathrm{a}$ \\
\hline fenpropathrin & 15,00 & $9,00 \pm 0,44 \mathrm{ab}$ & $8,80 \pm 0,37 \mathrm{ab}$ & $9,60 \pm 0,24 \mathrm{a}$ & $10,00 \pm 0,00 \mathrm{a}$ & $10,00 \pm 0,00 \mathrm{a}$ & $10,00 \pm 0,00 \mathrm{a}$ & $10,00 \pm 0,00 \mathrm{a}$ & $10,00 \pm 0,0 \mathrm{a}$ \\
\hline óxido fenbutatin & 40,00 & $9,80 \pm 0,20 \mathrm{a}$ & $10,00 \pm 0,00 \mathrm{a}$ & $10,00 \pm 0,00 \mathrm{a}$ & $10,00 \pm 0,00 \mathrm{a}$ & $10,00 \pm 0,00 \mathrm{a}$ & $10,00 \pm 0,00 \mathrm{a}$ & $10,00 \pm 0,00 \mathrm{a}$ & $10,00 \pm 0,0 \mathrm{a}$ \\
\hline propargite & 72,00 & $9,00 \pm 0,31 \mathrm{ab}$ & $9,40 \pm 0,24 \mathrm{ab}$ & $10,00 \pm 0,00 \mathrm{a}$ & $10,00 \pm 0,00 \mathrm{a}$ & $10,00 \pm 0,00 \mathrm{a}$ & $10,00 \pm 0,00 \mathrm{a}$ & $10,00 \pm 0,00 \mathrm{a}$ & $10,00 \pm 0,0 \mathrm{a}$ \\
\hline pyridaben & 15,00 & $0,00 \pm 0,00 \mathrm{~d}$ & $0,00 \pm 0,00 \mathrm{~d}$ & $0,00 \pm 0,00 \mathrm{~b}$ & $0,20 \pm 0,20 \mathrm{c}$ & $2,40 \pm 0,40 \mathrm{c}$ & $7,60 \pm 1,26 \mathrm{~b}$ & $10,00 \pm 0,00 \mathrm{a}$ & $10,00 \pm 0,0 \mathrm{a}$ \\
\hline Testemunha & - & $10,00 \pm 0,00 \mathrm{a}$ & $9,80 \pm 0,25 \mathrm{a}$ & $10,00 \pm 0,00 \mathrm{a}$ & $10,00 \pm 0,00 \mathrm{a}$ & $9,80 \pm 0,25 \mathrm{a}$ & $10,00 \pm 0,00 \mathrm{a}$ & $10,00 \pm 0,00 \mathrm{a}$ & $10,00 \pm 0,0 \mathrm{a}$ \\
\hline $\mathrm{CV}(\%)$ & - & 5,27 & 5,75 & 5,59 & 5,45 & 4,73 & 4,15 & 4,15 & 1,25 \\
\hline
\end{tabular}

TABELA 3 - Toxicidade residual de agrotóxicos sobre Neoseiulus californicus, 72 horas após a transferência de ácaros para folhas de citros tratadas em diferentes períodos, da aplicação à transferência: Porcentagem de redução populacional.

\begin{tabular}{|c|c|c|c|c|c|c|c|c|c|}
\hline \multirow[t]{2}{*}{ Nome técnico } & \multirow{2}{*}{$\begin{array}{c}\text { Dosagem } \\
\text { g de i.a./100 } \\
\text { L }\end{array}$} & \multicolumn{8}{|c|}{ Período após a aplicação } \\
\hline & & 2 horas & 1 dia & $3 \mathrm{dia}$ & 5 dia & 7 dia & $10 \mathrm{dia}$ & $14 \mathrm{dia}$ & $21 \mathrm{dia}$ \\
\hline abamectin & 0,54 & $29,8^{1}$ & 0,0 & 0,0 & 0,0 & 0,0 & 0,0 & 0,0 & 0,0 \\
\hline acrinathrin & 0,50 & 6,2 & 4,2 & 0,0 & 0,0 & 0,0 & 0,0 & 0,0 & 0,0 \\
\hline azocyclotin & 25,00 & 24,0 & 32,0 & 2,0 & 0,0 & 0,0 & 0,0 & 0,0 & 0,0 \\
\hline cyhexatin & 25,00 & 34,1 & 18,8 & 6,9 & 0,0 & 0,0 & 0,0 & 0,0 & 0,0 \\
\hline chlorfenapyr & 15,00 & 100,0 & 95,8 & 96,0 & 96,0 & 89,8 & 77,1 & 40,5 & 10,5 \\
\hline deltamethrin & 1,25 & 4,2 & 2,2 & 0,0 & 0,0 & 0,0 & 0,0 & 0,0 & 0,0 \\
\hline dicofol & 37,00 & 100,0 & 93,8 & 68,0 & 68,0 & 21,0 & 17,1 & 2,0 & 0,0 \\
\hline dinocap & 18,45 & 6,0 & 6,2 & 0,0 & 0,0 & 0,0 & 0,0 & 0,0 & 0,0 \\
\hline enxofre & 400,00 & 0,1 & 0,0 & 0,0 & 0,0 & 0,0 & 0,0 & 0,0 & 0,0 \\
\hline fenpropathrin & 15,00 & 8,2 & 10,3 & 0,0 & 0,0 & 0,0 & 0,0 & 0,0 & 0,0 \\
\hline óxido de fenbutatin & 40,00 & 2,0 & 0,0 & 0,0 & 0,0 & 0,0 & 0,0 & 0,0 & 0,0 \\
\hline propargite & 72,00 & 6,3 & 0,0 & 0,0 & 0,0 & 0,0 & 0,0 & 0,0 & 0,0 \\
\hline pyridaben & 15,00 & 100,0 & 100,0 & 98,0 & 75,0 & 75,0 & 17,4 & 0,0 & 0,0 \\
\hline
\end{tabular}

${ }^{1}$ Calculada pela fórmula de Henderson \& Tilton (1955). 
Aos 7 dias após a aplicação, dicofol apresentou redução na toxicidade a $N$. californicus, para níveis inferiores a $25 \%$, enquanto chlorfenapyr e pyridaben se mantinham prejudiciais ao fitoseídeo, causando mais de $75 \%$ de mortalidade (Tabela 2).

Clorfenapyr causou alta mortalidade de Neoseiulus californicus dez dias após a aplicação; todavia, nos tratamentos com dicofol e pyridaben, a mortalidade foi reduzida (d" 17,4\%). Entretanto, o chlorfenapyr acarretou mortalidades de 40,5 e 10,5\% a $N$. californicus, aos 14 e 21 dias após a aplicação, respectivamente.

A redução da mortalidade acarretada pelos vários produtos à medida que o período entre a aplicação e a transferência aumentava, ou seja, de duas horas para 21 dias, foi mais lenta nos tratamentos com chlorfenapyr, pyridaben e dicofol (Tabela 3).

Assim sendo, para essa população de $N$. californicus, as liberações poderiam ser realizadas três dias após a aplicação para todos os produtos, com exceção do cyhexatin, dicofol, pyridaben e chlorfenapyr, com pouca probabilidade de ocorrer mortalidade significativa de adultos. Para cyhexatin, dicofol, pyridaben e chlorfenapyr, as liberações deveriam ser realizadas aos 5; 14; 14 e 21 dias após a aplicação, respectivamente.

Ressalta-se ainda que os resultados obtidos neste trabalho, complementados com trabalhos de campo, contribuirão para uma escolha mais adequada dos agrotóxicos a serem utilizados em programas de manejo integrado, em pomares de citros, nos quais $N$. californicus esteja presente, ou naqueles em que o predador venha a ser liberado. A manutenção desse fitoseídeo nos pomares cítricos pode propiciar maior eficiência de controle das populações dos ácaros fitófagos, minimizando as aplicações de acaricidas e reduzindo o custo final da produção.

\section{CONCLUSÕES}

1. Resíduos dos produtos acrinathrin, deltamethrin, dinocap, enxofre, fenpropathrin, propargite e óxido de fenbutatin, em folhas de citros, não causaram mortalidades significativas em adultos de $N$. californicus.

2. Adultos do ácaro predador expostos aos resíduos de abamectin, azocyclotin e cyhexatin, com duas horas de idade, apresentaram mortalidades entre 24 e $35 \%$.

3. Os produtos mais tóxicos ao predador foram dicofol, pyridaben e chlorfenapyr, causando $100 \%$ de mortalidade em ácaros expostos aos resíduos desses acaricidas, com duas horas de idade.

4. O resíduo de abamectin provocou mortalidade significativa em $N$. californicus, por um período inferior a um dia.

5. Os resíduos dos acaricidas azocyclotin, cyhexatin, dicofol, pyridaben e chlorfenapyr provocaram mortalidades significativas em adultos de $N$. californicus, por períodos de 1 ; $1 ; 10 ; 10$ e 21 dias, respectivamente.

\section{REFERÊNCIAS}

ANDREI, E. (Ed.) Compêndio de defensivos agrícolas. 6. ed. São Paulo: Andrei, 1999. 672p.

CROFT, B.A.; M. WHALON, E. Selective toxicity of pyrethroid insecticides to arthropod natural enemies and pest of agricultural crops. Entomophaga, Paris, v.27, p.3$21,1982$.

HENDERSON, C.F.; TILTON, E.W. Tests with acaricides against the brown wheat mite. Journal Economic Entomology, Lanham, v.63, p.1536- 539, 1955.

KNIGHT, A.L.; BEERS, E.H.; HOYT, S.C.; RIEDL, H. Acaricide bioassay with spider mites (Acari: Tetranychidae) on pome fruits: evaluation of methods and selection of discrimination concentrations for resistance monitoring. Journal Economic Entomology, Lanham, v.83, n.5, p.1752-1760, 1990.

KOMATSU, S.S.; NAKANO, O. Estudos visando ao manejo do ácaro da leprose em citros através do ácaro predador Euseius concordis (Acari: Phytoseiidae). Laranja, Cordeiropólis, v.9, n.1, p.125-146, 1988.

MALEZIEUX, S.; LAPCHIN, L.; PRALAVORIO, M.; MOULIN, J.C.; FOURNIER, D. Toxicity of pesticide residues to beneficial arthropod, Phytoseiulus persimilis (Acari: Phytoseiidae). Journal Economic Entomology, Lanham, v.85, p.2077-2081, 1992.

McMURTRY, J.A.; CROFT, B.A. Life styles of phytoseiid mites and their roles as biological control agents. Annual Review of Entomology, Palo Alto, v.42, p.291321, 1997.

MONTEIRO, L.B. Manejo integrado de Panonychus ulmi em macieira. Primeiras experiências com a introdução de Neoseiulus californicus. Revista Brasileira de Fruticultura, Jaboticabal, v.16, p.46-53, 1994.

MONTEIRO, L.B. Manejo integrado de pragas em macieira no Rio Grande do Sul II. Uso de Neoseiulus californicus para controle de Panonychus ulmi. Revista Brasileira de Fruticultura, Jaboticabal, v.24, n.2, p.395-405, 2002.

MORAES, G.J. de; McMURTRY. J.A.;DENMARK, H.A. A catalog of the mite family Phytoseiidae: references to taxonomy, synonymy, distribution and habitat. Brasília: EMBRAPADDT,1986. 553p.

MORAES, G.J. de. Perspectivas para uso de predadores no controle de ácaros fitófagos no Brasil. Pesquisa Agropecuária Brasileira, Brasília, v.27, p.263-270, 1992.

MORAES, G.J. de; McMURTRY, J.A.; DENMARK, H.A.; CAMPOS, C.B. A revised catalog of mite family Phytoseiidae. Zootaxa, Aukland, v.434, p.1-494,2004.

MORSE, J.G.; BELLOWS JR., T.S.; GASTON, L.K.; IWATA, Y. Residual toxicity of acaricides to three beneficial species on California citrus. Journal Economic Entomology. Lanham, v.80, p.953-960, 1987.

REIS, P.R.; CHIAVEGATO. L.G.; MORAES, G.J. de; ALVES, E.B.; SOUSA, E.O. Seletividade de agroquímicos ao ácaro predador Iphiseiodes zuluagai Denmark \& Muma (Acari: Phytoseiidae). Anais da Sociedade Entomológica do Brasil, Londrina, v.27, n.2, p.265-274. 1998. 
REIS, P.R.; SOUSA, E.O.; ALVES, E.B. Seletividade de produtos fitossanitários ao ácaro predador Euseius alatus De Leon( Acari: Phytoseiidae). Revista Brasileira de Fruticultura, Jaboticabal, v.21, n.3, p.350355, 1999.

SATO, M.E.; RAGA, A.; CERÁVOLO, L.C.; CEZÁRIO, A.C; ROSSI, A. Efeito da utilização de acaricidas em citros, sobre a população de Brevipalpus phoenicis (Geijskes, 1939) (Acari: Tenuipalpidae) e ácaros predadores (família Phytoseiidae). Scientia Agricola, Piracicaba, v.52, n.2, p.282$286,1995$.

SATO, M.E.; RAGA, A.; CERÁVOLO, L.C.; ROSSI, A.C.; SOUZA FILHO, M.F. de. Toxicidade residual de acaricidas a Iphiseiodes zuluagai Denmark \& Muma, 1972 (Acari: Phytoseiidae). Arquivos do Instituto Biológico, São Paulo, v.63, p.15-19, 1996.

SATO, M.E.; RAGA, A. Divulgação técnica: Ácaro da Leprose. O Biologico, São Paulo, v.63, p.15-19, 1998

VAN DE VRIE, M.; McMURTRY, J.A.; HUFFAKER, C.B. Ecology of tetranychid mites and their natural enemies: A review. III. Biology, ecology, and pest status, and host-plant relations of tetranychids. Hilgardia, Berkeley, v.41, p.387-403, 1972.

YAMAMOTO, P.T.; PINTO, A.S.; PAIVA, P.E.B.; GRAVENA, S. Seletividade de agrotóxicos aos inimigos naturais de pragas dos citros. Laranja, Cordeirópolis, v.13, p.709-755, 1992.

YAMAMOTO, P.T.; PINTO, A.S.; PAIVA, P.E.B.; GRAVENA, S. Seletividade de acaricidas a inimigos naturais em citros. In: OLIVEIRA, C.A.L. de; DONADIO, L C. Leprose dos citros. Jaboticabal: FUNEP, 1995. p.159-170.

ZACHARDA, M.; HLÙCHY, M. Long-term residual efficacy of commercial formulations of pesticides to Typhlodromus pyri Scheuten (Acari: Phytoseiidae) inhabiting commercial vineyards. Experimental and Applied Acarology, Amsterdam, v.13, p.27-40, 1991. 